\title{
Pseudospin Anisotropy Classification of Quantum Hall Ferromagnets
}

\author{
T. Jungwirth ${ }^{1,2}$ and A.H. MacDonald ${ }^{1}$ \\ ${ }^{1}$ Department of Physics, Indiana University, Bloomington, Indiana 47405 \\ ${ }^{2}$ Institute of Physics ASCR, Cukrovarnická 10, 162 00 Praha 6, Czech Republic
}

(November 11, 2018)

\begin{abstract}
Broken symmetry ground states with uniform electron density are common in quantum Hall systems when two Landau levels simultaneously approach the chemical potential at integer filling factor $\nu$. The close analogy between these two-dimensional electron system states and conventional itinerant electron ferromagnets can be emphasized by using a pseudospin label to distinguish the two Landau levels. As in conventional ferromagnets, the evolution of the system's state as external field parameters are varied is expected to be sensitive to the dependence of ground state energy on pseudospin orientation. We discuss the predictions of Hartree-Fock theory for the dependence of the sign and magnitude of the pseudospin anisotropy energy on the nature of the crossing Landau levels. We build up a classification scheme for quantum Hall ferromagnets that applies for single layer and bilayer systems with two aligned Landau levels distinguished by any combination of real-spin, orbitradius, or growth direction degree-of-freedom quantum numbers. The possibility of in-situ tuning between easy-axis and easy-plane quantum Hall ferromagnets is discussed for biased bilayer systems with total filling factors $\nu=3$ or $\nu=4$. Detailed predictions are made for the bias dependence of pseudospin reversal properties in $\nu=3$ bilayer systems.
\end{abstract}

73.40.Hm, 75.10.Lp, 75.30.Gw

\section{INTRODUCTION}

Studies of magnetic phenomena in semiconductors have opened fruitful new ways to explore the subtleties of quantum magnetism. In quantum Hall samples, the tunability of semiconductor electronic systems and the quantization of single-particle energies into macroscopically degenerate Landau levels (LLs) combine to open up a rich and varied phenomenology. The effective zero width of electronic energy bands enhances the role of inter-particle interactions 1 and can frequently lead to the formation of ordered many-particle ground states, including ferromagnetic ones.

Most studies of quantum Hall ferromagnets have focused on spontaneous spin-alignment in a singlelayer two-dimensional (2D) electron system at LL filling factor $\nu=1$. In this case it turns out that electronelectron interactions favor fully aligned electron spinf even in the limit of vanishingly small Zeeman coupling and the ferromagnetic ground state of the system is described exactly by Hartree-Fock (HF) theory. Because of the near spin-independence of the Coulomb interaction, the $\nu=1$ single layer QHF is a Heisenberg-like isotropic two-dimensional ferromagnet. One of the unique properties of this simple itinerant electron ferromagnet is that its ipstantons 3 (Skyrmions) carry charge and can be observed 1 in the ground states at filling factors slightly deviating from 1 .

The notion of the QHF can be generalized however. It turns out that, at least according to HF theory, broken symmetry ground states occur at integer filling factors in quantum Hall systems any time two or more valence LLs are degenerate and the number of electrons is sufficient to fill only some of the LLs. In effect, electrons in the ordered state occupy spontaneously generated LLs that are linear combinations of the single-particle levels chosen to minimize the electron interaction energy.

The simplest example of a pseudospin QHF obtains at $\nu=1$ in balanced bilayer 2D systems where the singleparticle LLs in the twe-layers are degenerate. In the ordered ground state2 5 . 6 the electrons occupy a LL which is a linear combination of the isolated layer levels, forming a state with spontaneous inter-layer phase coherence. Recently, Josephson-like behavior seent in 2D-to-2D tunneling spectroscopy studies of bilayer systems has provided a direct manifestation of collective behavior generated by this broken symmetry. In $\nu=1$ bilayer systems, the broken symmetry state minimizes the Hartree energy cost by distributing charge equally between layers and gives up part of the intra-layer exchange energy while gaining more in inter-layer exchange energy. Unlike the single layer $\nu=1 \mathrm{QHF}$, the ordered HF ground state is not exact in this case, and the order predicted by HF theory can be destroyed. With decreasing interlayer exchange energy quantum fluctuations around the mean-field ordered state become more important and for layer separations larger than approximately two magnetic lengths fluctuations destroy the spontaneous coherence. The corresponding order-disorder quantum phase transition has been observed experimentally. 6 In $\nu=1$ bilayer QHFs, it is the layer degree of freedom which is represented as a pseudospin- $1 / 2$. With this mapping the phase coherent state is equivalent to a spin- $1 / 2$ easy-plane ferromagnet. Finite temperature Kosterlitz-Thouless phase 
transition 1 , continuous quantum phase transition induced by in-plane magnetic fieldB, and macroscopic collective transport effects are are among the remarkable phenomena which have been studiedon bilayer QHEs.

Recent experiments in single layer 10 and bilayer $112 \mathrm{D}$ systems at even-integer filling factors have further enlarged the field of quantum Hall ferromagnetism. It has been show that easy-axis ferromagnetic ground states can occur 10 at higher filling factors when LLs with different orbit radius quantum numbers are brought close to alignment 12,10 In HF language, easy-axis anisotropy means that many-body states with either of the two aligned LLs completely filled and the other empty is energetically more favorable than the coherent superposition state. The easy-axis pseudospin anisotropy occurs in this case because intra LL exchange is stronger than exchange between particles from LLs with different orkit radius quantum numbers. Transport measurement, 11,13 have demonstrated that easy-axis QHFs exhibit hysteresis with a complicated phenomenology, presumably associated with an interplay between disorder and domainmorphology similar to that in conventional thin film magnets.

In this paper we classify QHFs according to their pseudospin anisotropy energies as either isotropic, easy-axis, or easy-plane systems. We report on a HF based analysis which predicts how the class of broken symmetry ground state depends on the nature of the crossing LLs. In some cases, competing effects allow the anisotropy energy to be tuned continuously generating a zero-temperature quantum phase transition between different classes of states. We consider only cases where no more than two LLs are nearly degenerate and the number of electrons is sufficient to occupy one of them. We will always assume that lower energy LLs, if present, are completely full and higher energy LLs are completely empty; coupling to these remote LLs can usually be treated perturbatively if necessary, although we do not do so explicitly here. An important example of an instance in which more than two LLs are close to degeneracy pertains in double-layer systems with weak tunneling and weak Zeeman coupling; 44.15 we do not treat this or other more complex cases with many degenerate LLs in this paper. In Section II we precisely define the pseudospin language we use in which one of the LLs is referred to as the pseudospin-up state and the other LL as the pseudospindown state. Since we assume that the magnetic field is perpendicular to the $2 \mathrm{D}$ electron layer, growth direction and in-plane degrees of freedom decouple. The pseudospin quantum number then subsumes real-spin, orbit radius, and growth direction (subband) degrees of freedom. To make the discussion more transparent we concentrate on a system consisting of two nearby infinitely narrow 2D layers, the simplest model which has a nontrivial growth direction degree of freedom. Comments are made throughout the text about realistic samples with more complicated geometries. In Section III we derive a general expression for the HF ground state energy in the pseudospin ferromagnetic state. Section IV summarizes the rather cumbersome evaluation of Coulomb interaction matrix elements. Readers not interested in technical details of the calculation are encouraged to skip to Section V where we present our conclusions concerning pseudospin magnetic anisotropy of single layer and bilayer QHFs. This section includes phase diagrams which show the regimes of physically tunable parameters with easy-axis and easy-plane anisotropies. Symmetry breaking fields and the dynamics of pseudospin reversal are discussed in Section VI. Finally, we conclude in Section VII with a brief summary of the main results of our paper.

\section{PSEUDOSPIN REPRESENTATION}

The pseudospin language was introduced 0 to the description of broken symmetry states in the quantum Hall regime, in order to draw on the analogy between double quantum well systems at $\nu=1$ and $2 \mathrm{D}$ ferromagnets. In this work the pseudospin degree of freedom represented the layer index of a bilayer system. Here we allow the pseudospin index to have a more general meaning. To establish terminology, it is useful to recall the singleparticle spectrum of a bilayer $2 \mathrm{D}$ system subject to a perpendicular magnetic field. Quantum well subbands of individual layers can be mixed by interlayer tunneling and shifted by the application of a bias potential. We limit our attention to the usual case where only the lowest electric subband of either quantum well is occupied and for explicit calculations use a zero-width quantum well model. Allowing for external bias and for tunneling between the wells (see Fig. 1), the bilayer subband wavefunctions of the zero-width model are

$$
\lambda_{ \pm 1}(z)=\frac{1}{\sqrt{2}}\left[\left(1 \mp r_{\Delta}\right)^{1 / 2} \delta(z) \pm\left(1 \pm r_{\Delta}\right)^{1 / 2} \delta(z-d)\right]
$$

where $r_{\Delta}=\Delta_{V} /\left(\Delta_{V}^{2}+\Delta_{t}^{2}\right)^{1 / 2}, \Delta_{V}$ is the bias potential, $\Delta_{t}$ the tunneling gap at zero bias, and $d$ is the layer separation. To account for specific experimental samples, finite width effects can be incorporated by replacing the wavefunctions (11) with electric subband wavefunctions calculated using the self-consistent local-spindensity-approximation (LSDA) model. 16

In the Landau gauge the wavefunctions in the 2D plane take a form $\phi_{n, s, k}(x) \exp (i k y) / \sqrt{L_{y}}$, where

$$
\begin{aligned}
\phi_{n, s, k}(x) & =\left[\pi \ell^{2} 2^{2 n}(n !)^{2}\right]^{-1 / 4} H_{n}\left(\frac{x-\ell^{2} k}{\ell}\right) \\
& \times \exp \left[-\frac{\left(x-\ell^{2} k\right)^{2}}{2 \ell^{2}}\right]
\end{aligned}
$$

$k$ is the wavevector label which distinguishes states within a LL, $n=0,1, \ldots$ is the orbit radius quantum number, $\ell$ is the magnetic length, and we have also explicitly 
included the real-spin, $s= \pm 1 / 2$, degree of freedom. The single-particle energy spectrum consists of discrete LLs

$$
E_{\xi, n, s}=-\frac{\xi}{2}\left(\Delta_{V}^{2}+\Delta_{t}^{2}\right)^{1 / 2}+\hbar \omega_{c}\left(n+\frac{1}{2}\right)-s|g| \mu_{B} B,
$$

where $\omega_{c}$ is the cyclotron frequency and the last term is the real-spin Zeeman coupling. Each LL has a macroscopic degeneracy with the number of orbital states per level $N_{\phi}=A B / \Phi_{0}$, where $A$ is the system area, $B$ is the field strength, and $\Phi_{0}$ is the magnetic flux quantum.

The many-body broken symmetry states we study in the following sections occur when two LLs are brought close to alignment while remaining sufficiently separated from other LLs. In our calculations, each LL can have one of two possible subband indices, one of two possible spin indices, and any value for the $2 \mathrm{D}$ cyclotron orbit kinetic energy index. The two crossing LLs can differ in any or all of these labels. We label one of the two levels as the pseudospin-up $(\sigma=\uparrow)$ state and the other level as the pseudospin down $(\sigma=\downarrow)$ state. We truncate the single-particle Hilbert space by ignoring higher LLs and introducing effective one-body fields that account for the effect of electrons in lower LLs on the two pseudospin states. Within this model the set of single-particle states reduces to following wavefunctions

$$
\psi_{\sigma, k}(\vec{r})=\lambda_{\xi(\sigma)}(z) \phi_{n(\sigma), s(\sigma), k}(x) \frac{\exp (i k y)}{\sqrt{L_{y}}} .
$$

A particle with the pseudospin oriented along a general unit vector $\hat{m}=(\sin \theta \cos \varphi, \sin \theta \sin \varphi, \cos \theta)$ is described by

$$
\psi_{\hat{m}, k}(\vec{r})=\cos \left(\frac{\theta}{2}\right) \psi_{\uparrow, k}(\vec{r})+\sin \left(\frac{\theta}{2}\right) e^{i \varphi} \psi_{\downarrow, k}(\vec{r}) .
$$

\section{MANY-BODY HAMILTONIAN AND HF TOTAL ENERGY}

In the HF approximation, the QHF has a single Slater determinant state with the same pseudospin orientation for every orbital $k$. In this section we derive general expressions for the dependence of the many-electron state energy on pseudospin orientation. It is convenient to express the many-body Hamiltonian using Pauli spinmatrices $\tau_{x}, \tau_{y}$, and $\tau_{z}$ and the $2 \times 2$ identity matrix which we label $\tau_{\mathbf{1}}$. In this representation the Hamiltonian reads

$$
\begin{aligned}
H & =-\sum_{i=\mathbf{1}, x, y, z} \sum_{k=1}^{N_{\phi}} \sum_{\alpha, \alpha^{\prime}=1}^{2} b_{i} \tau_{i}^{\alpha^{\prime}, \alpha} c_{\sigma\left(\alpha^{\prime}\right), k}^{\dagger} c_{\sigma(\alpha), k} \\
& +\frac{1}{2} \sum_{i, j=\mathbf{1}, x, y, z} \sum_{\substack{k_{1}, k_{1}^{\prime}, k_{2}, k_{2}^{\prime}=1}}^{N_{\phi}} \sum_{\substack{\alpha_{1}, \alpha_{1}^{\prime}, \alpha_{2}, \alpha_{2}^{\prime}=1}}^{2} W_{i, j}^{k_{1}^{\prime}, k_{2}^{\prime}, k_{1}, k_{2}} \tau_{i}^{\alpha_{1}^{\prime}, \alpha_{1}} \tau_{j}^{\alpha_{2}^{\prime}, \alpha_{2}} \\
& \times c_{\sigma\left(\alpha_{1}^{\prime}\right), k_{1}^{\prime}}^{\dagger} c_{\sigma\left(\alpha_{2}^{\prime}\right), k_{2}^{\prime}}^{\dagger} c_{\sigma\left(\alpha_{2}\right), k_{2}} c_{\sigma\left(\alpha_{1}\right), k_{1}},
\end{aligned}
$$

where $\sigma(1)=\uparrow$ and $\sigma(2)=\downarrow$. The one-body terms $b_{i}$ include, in general, the external bias potential, tunneling, cyclotron and Zeeman energies and also the mean-fields from interactions with electrons in the frozen LLs lying below the $\sigma=\uparrow$ and $\downarrow$ levels. We will give an explicit expression for $b_{i}$ in section VI. Here and in the following two sections, we concentrate on the two-body terms in the Hamiltonian (6).

The potentials $W_{i, j}$ represent different combinations of Coulomb interaction matrix elements, $V_{\sigma_{1}^{\prime}, \sigma_{2}^{\prime}, \sigma_{1}, \sigma_{2}}$, of the single-particle pseudospin states

$$
\begin{aligned}
V_{\sigma_{1}^{\prime}, \sigma_{2}^{\prime}, \sigma_{1}, \sigma_{2}}^{k_{1}^{\prime}, k_{2}^{\prime}, k_{2}, k_{2}} & =\int d^{3} \vec{r}_{1} \int d^{3} \vec{r}_{2} \psi_{\sigma_{1}^{\prime}, k_{1}^{\prime}}^{*}\left(\vec{r}_{1}\right) \psi_{\sigma_{2}^{\prime}, k_{2}^{\prime}}^{*}\left(\vec{r}_{2}\right) \\
& \times \frac{e^{2}}{\epsilon\left|\vec{r}_{1}-\vec{r}_{2}\right|} \psi_{\sigma_{1}, k_{1}}\left(\vec{r}_{1}\right) \psi_{\sigma_{2}, k_{2}}\left(\vec{r}_{2}\right)
\end{aligned}
$$

General expressions for the pseudospin dependent interactions $W_{i, j}$ are given in Table $\rrbracket$ in terms of the following matrix element combinations:

$$
\begin{aligned}
B_{1}^{ \pm} & =\frac{1}{4}\left(V_{\uparrow, \uparrow, \uparrow, \uparrow} \pm V_{\downarrow, \downarrow, \downarrow, \downarrow}\right), B_{2}^{ \pm}=\frac{1}{4}\left(V_{\uparrow, \downarrow, \uparrow, \downarrow} \pm V_{\downarrow, \uparrow, \downarrow, \uparrow}\right) \\
B_{3}^{ \pm} & =\frac{1}{4}\left(V_{\uparrow, \downarrow, \downarrow, \uparrow} \pm V_{\downarrow, \uparrow, \uparrow, \downarrow}\right), B_{4}^{ \pm}=\frac{1}{4}\left(V_{\uparrow, \uparrow, \downarrow, \downarrow} \pm V_{\downarrow, \downarrow, \uparrow, \uparrow}\right) \\
B_{5}^{ \pm} & =\frac{1}{4}\left(V_{\uparrow, \uparrow, \uparrow, \downarrow} \pm V_{\uparrow, \downarrow, \uparrow, \uparrow}\right), B_{6}^{ \pm}=\frac{1}{4}\left(V_{\downarrow, \uparrow, \downarrow, \downarrow} \pm V_{\downarrow, \downarrow, \downarrow, \uparrow}\right) \\
B_{7}^{ \pm} & =\frac{1}{4}\left(V_{\uparrow, \uparrow, \downarrow, \uparrow} \pm V_{\downarrow, \uparrow, \uparrow, \uparrow}\right), B_{8}^{ \pm}=\frac{1}{4}\left(V_{\uparrow, \downarrow, \downarrow, \downarrow} \pm V_{\downarrow, \downarrow, \uparrow, \downarrow}\right)
\end{aligned}
$$

In (8) we have omitted orbital guiding center indices for simplicity.

The many-electron state with pseudospin orientation $\hat{m}$ is $|\Psi[\hat{m}]\rangle=\prod_{k=1}^{N_{\phi}} c_{\hat{m}, k}^{\dagger}|0\rangle$, where $c_{\hat{m}, k}^{\dagger}$ creates the single-particle state whose wavefunction is given in Eq. (5). We find that

$$
\begin{aligned}
e_{H F}(\hat{m}) & \equiv \frac{\langle\Psi[\hat{m}]|H| \Psi[\hat{m}]\rangle}{N_{\phi}} \\
& =-\sum_{i=x, y, z}\left(b_{i}-\frac{1}{2} U_{\mathbf{1}, i}-\frac{1}{2} U_{i, \mathbf{1}}\right) m_{i} \\
& +\frac{1}{2} \sum_{i, j=x, y, z} U_{i, j} m_{i} m_{j},
\end{aligned}
$$

where

$$
U_{i, j}=\frac{1}{N_{\phi}} \sum_{k_{1}, k_{2}=1}^{N_{\phi}}\left(W_{i, j}^{k_{1}, k_{2}, k_{1}, k_{2}}-W_{i, j}^{k_{2}, k_{1}, k_{1}, k_{2}}\right)
$$

has direct and exchange contributions. Eq. (9) is the most general form for the HF energy of a pseudospin- $1 / 2$ QHF. The magnetic anisotropy of the particular QHF system is governed by the terms in (9) that are quadratic in the pseudospin magnetization $m_{i}$. The values of the coefficients $U_{i, j}$ depend on the nature of the crossing LLs and and can result in isotropic, easy-plane, or easy-axis quantum Hall ferromagnetism. 


\section{PSEUDOSPIN MATRIX ELEMENTS OF THE COULOMB INTERACTION}

This section contains the derivation of explicit expressions for the anisotropy energy coefficients $U_{i, j}$, assuming the zero-width quantum well model wavefunctions (11). Using the Fourier representation of the Coulomb interaction we write the pseudospin matrix elements as

$$
\begin{array}{r}
\quad V_{\sigma_{1}^{\prime}, \sigma_{2}^{\prime}, \sigma_{1}, \sigma_{2}}^{k_{1}^{\prime}, k^{\prime}, k_{1}, k_{2}}=\frac{1}{A} \sum_{\vec{q}} \delta_{q_{y}, k_{1}^{\prime}-k_{1}} \delta_{-q_{y}, k_{2}^{\prime}-k_{2}} \\
\times e^{i q_{x}\left(k_{1}^{\prime}+k_{1}\right) / 2} e^{-i q_{x}\left(k_{2}^{\prime}+k_{2}\right) / 2} v_{\sigma_{1}^{\prime}, \sigma_{2}^{\prime}, \sigma_{1}, \sigma_{2}}(\vec{q})
\end{array}
$$

and hence

$$
\begin{aligned}
& \frac{1}{N_{\phi}} \sum_{k_{1}, k_{2}=1}^{N_{\phi}}\left(V_{\sigma_{1}^{\prime}, \sigma_{2}^{\prime}, \sigma_{1}, \sigma_{2}}^{k_{1}, k_{2}, k_{1}, k_{2}}-V_{\sigma_{1}^{\prime}, \sigma_{2}^{\prime}, \sigma_{1}, \sigma_{2}}^{k_{2}, k_{1}, k_{1}, k_{2}}\right)= \\
& \frac{N_{\phi}}{A} v_{\sigma_{1}^{\prime}, \sigma_{2}^{\prime}, \sigma_{1}, \sigma_{2}}(0)-\frac{1}{A} \sum_{\vec{q}} v_{\sigma_{1}^{\prime}, \sigma_{2}^{\prime}, \sigma_{1}, \sigma_{2}}(\vec{q})
\end{aligned}
$$

In the following we take $\ell$ as the unit of length and $e^{2} / \epsilon \ell$ as the unit of energy. Then $N_{\phi} / A=1 / 2 \pi$ and Eq. (12) can be rewritten in the more transparent form

$$
\begin{aligned}
& \frac{1}{N_{\phi}} \sum_{k_{1}, k_{2}=1}^{N_{\phi}}\left(V_{\sigma_{1}^{\prime}, \sigma_{2}^{\prime}, \sigma_{1}, \sigma_{2}}^{k_{1}, k_{2}, k_{1}, k_{2}}-V_{\sigma_{1}^{\prime}, \sigma_{2}^{\prime}, \sigma_{1}, \sigma_{2}}^{k_{2}, k_{1}, k_{1}, k_{2}}\right)= \\
& \int \frac{d^{2} \vec{q}}{(2 \pi)^{2}} e^{-q^{2} / 2}\left[v_{\sigma_{1}^{\prime}, \sigma_{2}^{\prime}, \sigma_{1}, \sigma_{2}}(0)-v_{\sigma_{1}^{\prime}, \sigma_{2}^{\prime}, \sigma_{1}, \sigma_{2}}(\vec{q})\right] .
\end{aligned}
$$

The first factor in square brackets in this equation originates from the Hartree contribution to the energy while the second factor originates from the exchange contribution.

In these equations $v_{\sigma_{1}^{\prime}, \sigma_{2}^{\prime}, \sigma_{1}, \sigma_{2}}(\vec{q})$ is a pseudospindependent effective 2D interaction which, because of the separability of the in-plane and out-of-plane degree-offreedom terms in the single electron Schroedinger equation, is the product of two factors: the subband factor

$$
\begin{aligned}
& v_{\sigma_{1}^{\prime}, \sigma_{2}^{\prime}, \sigma_{1}, \sigma_{2}}^{\Xi}(\vec{q})=\int_{-\infty}^{\infty} d z_{1} \int_{-\infty}^{\infty} d z_{2} e^{-q\left|z_{1}-z_{2}\right|} \\
\times & \lambda_{\xi\left(\sigma_{1}^{\prime}\right)}\left(z_{1}\right) \lambda_{\xi\left(\sigma_{2}^{\prime}\right)}\left(z_{2}\right) \lambda_{\xi\left(\sigma_{1}\right)}\left(z_{1}\right) \lambda_{\xi\left(\sigma_{2}\right)}\left(z_{2}\right)
\end{aligned}
$$

and the in-plane term

$$
\begin{aligned}
& v_{\sigma_{1}^{\prime}, \sigma_{2}^{\prime}, \sigma_{1}, \sigma_{2}}^{N}(\vec{q})=e^{q^{2} / 2} \\
\times & \int_{-\infty}^{\infty} d x_{1} \phi_{n\left(\sigma_{1}^{\prime}\right), s\left(\sigma_{1}^{\prime}\right), q_{y} / 2}\left(x_{1}\right) \phi_{n\left(\sigma_{1}\right), s\left(\sigma_{1}\right),-q_{y} / 2}\left(x_{1}\right) \\
\times & \int_{-\infty}^{\infty} d x_{2} \phi_{n\left(\sigma_{2}^{\prime}\right), s\left(\sigma_{2}^{\prime}\right),-q_{y} / 2}\left(x_{2}\right) \phi_{n\left(\sigma_{2}\right), s\left(\sigma_{2}\right), q_{y} / 2}\left(x_{2}\right)
\end{aligned}
$$

For a general sample geometry the subband factor (14) has to be calculated numerically using the selfconsistent LSDA wavefunctions. For our model bilayer system, however, we can obtain analytic expressions for $v_{\sigma_{1}^{\prime}, \sigma_{2}^{\prime}, \sigma_{1}, \sigma_{2}}^{\Xi}(\vec{q})$. In the case of $\xi(\sigma)=\xi(-\sigma)$, Eqs. (1) and (14) give

$$
v_{\sigma_{1}^{\prime}, \sigma_{2}^{\prime}, \sigma_{1}, \sigma_{2}}^{\Xi}=\frac{1}{2}\left[\left(1+r_{\Delta}^{2}\right)+\left(1-r_{\Delta}^{2}\right) e^{-d q}\right] .
$$

In the second case, i.e. $\xi(\sigma)=-\xi(-\sigma)$,

$$
\begin{aligned}
& v_{\sigma, \sigma, \sigma, \sigma}^{\Xi}=\frac{1}{2}\left[\left(1+r_{\Delta}^{2}\right)+\left(1-r_{\Delta}^{2}\right) e^{-d q}\right], \\
& v_{\sigma,-\sigma, \sigma,-\sigma}^{\Xi}=\frac{1}{2}\left[\left(1-r_{\Delta}^{2}\right)+\left(1+r_{\Delta}^{2}\right) e^{-d q}\right], \\
& v_{\sigma,-\sigma,-\sigma, \sigma}^{\Xi}=\frac{1}{2}\left(1-r_{\Delta}^{2}\right)\left(1-e^{-d q}\right),
\end{aligned}
$$

and

$$
\begin{aligned}
& v_{\sigma_{1}^{\prime}, \sigma_{2}^{\prime}, \sigma_{1}, \sigma_{2}}^{\Xi}=\eta \frac{r_{\Delta}}{2}\left(1-r_{\Delta}^{2}\right)^{1 / 2}\left(1-e^{-d q}\right) \\
& \text { if } \eta \equiv \frac{1}{2} \sum_{i=1}^{2}\left[\xi\left(\sigma_{i}^{\prime}\right)+\xi\left(\sigma_{i}\right)\right]= \pm 1 .
\end{aligned}
$$

For the in-plane factor in the effective interaction it is necessary to distinguish several cases. If the pseudospinup and pseudospin-down levels have the same realspin index and same orbit-radius quantum number, i.e. $s(\sigma)=s(-\sigma)$ and $n(\sigma)=n(-\sigma) \equiv n$, the effective interaction is independent of the pseudospin indices and we obtain from (2) and (15)

$$
v_{\sigma_{1}^{\prime}, \sigma_{2}^{\prime}, \sigma_{1}, \sigma_{2}}^{N}=\frac{2 \pi}{q}\left[L_{n}\left(q^{2} / 2\right)\right]^{2},
$$

where $L_{n}(x)$ is the Laguerre polynomial. For identical spins but different orbit-radius quantum numbers, i.e. $s(\sigma)=s(-\sigma)$ and $n(\sigma) \neq n(-\sigma)$, we define $n_{<} \equiv$ $\min [n(\sigma), n(-\sigma)]$ and $n_{>} \equiv \max [n(\sigma), n(-\sigma)]$. Then this factor in the effective interaction is

$$
\begin{aligned}
& v_{\sigma, \sigma, \sigma, \sigma}^{N}=\frac{2 \pi}{q}\left[L_{n(\sigma)}\left(q^{2} / 2\right)\right]^{2}, \\
& v_{\sigma,-\sigma, \sigma,-\sigma}^{N}=\frac{2 \pi}{q} L_{n(\sigma)}\left(q^{2} / 2\right) L_{n(-\sigma)}\left(q^{2} / 2\right), \\
& v_{\sigma,-\sigma,-\sigma, \sigma}^{N}=\frac{2 \pi}{q} \frac{n_{<} !}{n_{>} !}\left(\frac{q^{2}}{2}\right)^{n_{>}-n_{<}}\left[L_{n_{<}}^{n_{>}-n_{<}}\left(q^{2} / 2\right)\right]^{2}, \\
& \text { otherwise } \quad v_{\sigma_{1}^{\prime}, \sigma_{2}^{\prime}, \sigma_{1}, \sigma_{2}}^{N}=0 .
\end{aligned}
$$

If the two pseudospin LLs have opposite real-spins then only scattering processes that conserve pseudospin (and therefore also real-spin) at each vertex will contribute to the anisotropy energy. For $s(\sigma)=-s(-\sigma)$ and $n(\sigma)=$ $n(-\sigma) \equiv n$ we obtain

$$
\begin{aligned}
& v_{\sigma, \sigma, \sigma, \sigma}^{N}=v_{\sigma,-\sigma, \sigma,-\sigma}^{N}=\frac{2 \pi}{q}\left[L_{n}\left(q^{2} / 2\right)\right]^{2}, \\
& \text { otherwise } \quad v_{\sigma_{1}^{\prime}, \sigma_{2}^{\prime}, \sigma_{1}, \sigma_{2}}^{N}=0 .
\end{aligned}
$$


Finally if the pseudospin LLs have opposite real-spins and different orbit-radius quantum numbers, the in-plane factor in the effective interactions is

$$
\begin{aligned}
& v_{\sigma, \sigma, \sigma, \sigma}^{N}=\frac{2 \pi}{q}\left[L_{n(\sigma)}\left(q^{2} / 2\right)\right]^{2}, \\
& v_{\sigma,-\sigma, \sigma,-\sigma}^{N}=\frac{2 \pi}{q} L_{n(\sigma)}\left(q^{2} / 2\right) L_{n(-\sigma)}\left(q^{2} / 2\right), \\
& \text { otherwise } \quad v_{\sigma_{1}^{\prime}, \sigma_{2}^{\prime}, \sigma_{1}, \sigma_{2}}^{N}=0 .
\end{aligned}
$$

Eq. (14) for the subband factor and explicit expressions (18)-(21) for the in-plane factor in the effective interaction, together with Eqs. (13),(10), (8) and Table [1, provide a formal recipe to calculate the anisotropy coefficients $U_{i, j}$ for crossing LLs with any combination of quantum well subband, orbit radius and real-spin indeces. In the following section we use the explicit forms (17) and (16) for the subband factor to develope a pseudospin anisotropy classification scheme for our model bilayer system.

\section{MAGNETIC ANISOTROPY}

The nature of the anisotropy energy is of qualitative importance for two-dimensional ferromagnets, including QHFs. Systems with easy-axis anisotropy, i.e., discrete directions at which the energy of the ordered state is minimized, have long range order at finite temperature and phase transitions in the Ising universality class. Systems with easy-plane anisotropy, i.e., a continuum of coplanar pseudospin magnetization orientations at which the energy of the ordered state is minimized, do not have long-range order but do have Kosterlitz-Thouless phase transitions at a finite temperature. In the isotropic case, all directions of pseudospin magnetization have identical energy, only the ground state has long-range order, and there are no finite-temperature phase transitions. Nevertheless, magnetization correlations become extremely long at low temperatures. The objective of this work is to predict which class of QHF occurs for a particular pair of crossing LLs.

We start our analysis by considering pseudospin LLs that belong to the same subband, i.e. $\xi(\uparrow)=\xi(\downarrow)$. In this case, only LLs with opposite real-spins can be aligned. Two examples of QHFs falling into this category, total filling factor $\nu=1$ with $n(\uparrow)=n(\downarrow)=0$, and $\nu=2$ with $n(\uparrow)=1, n(\downarrow)=0$, are illustrated in Fig. 2. The cases of $n(\uparrow) \neq n(\downarrow)$ are realized when the ratio of the spinsplitting to LL separation is an integer. In GaAs, this ratio is only $\sim 1 / 60$ at perpendicular fields but can be tuned by tilting the magnetic field away from the normal to the 2D layer. For typical well widths orbital effects of the in-plane field, not included here, become important at the tilt angles where the coincidences of interest are realized and have to be accounted for 10 to obtain correct values of the pseudospin anisotropy energy coefficients
(10). However, recent work on AlAs quantum wells 17 and InSb quantum well 18 with large Zeeman couplings have made the situation we study below, which assumes perpendicular magnetic field, accessible.

When opposite spin LLs cross, Eqs. (20) and (21) imply that all interactions $B_{i}^{ \pm}$with $i>2$ in (8) vanish. Then the only non-zero anisotropy term is

$$
\begin{aligned}
U_{z, z} & =-\frac{1}{8} \int_{0}^{\infty} d q e^{-q^{2} / 2}\left[L_{n(\uparrow)}\left(q^{2} / 2\right)-L_{n(\downarrow)}\left(q^{2} / 2\right)\right]^{2} \\
& \times\left[\left(1+r_{\Delta}^{2}\right)+\left(1-r_{\Delta}^{2}\right) e^{-d q}\right] .
\end{aligned}
$$

Note that the Hartree energy contribution to anisotropy always vanishes when the crossing LLs share the same subband wavefunction. If the two pseudospin levels also have the same orbit-radius quantum number then Eq. (22) gives $U_{z, z}=0$ and the ferromagnetic state is isotropic. Physically, the result follows from the independence of the Coulomb interaction strength on real-spin. An important example of these isotropic QHFs occurs when $n(\uparrow)=n(\downarrow)=0$ and $r_{\Delta}=1$, i.e., there is no tunneling between layers This is the thoroughly studied singlelayer $\nu=1 \mathrm{QHF}$ - 4 for which the HF theory ground state happens to be exact. We remark that quantitative estimates based on the HF mean-field theory presented here require corrections to quantum fluctuation effects in cases when the ordered pseudospin moment direction is not a good quantum number. A detail understanding of these corrections is one challenge for future experimental and theoretical work on QHFs.

For $n(\uparrow) \neq n(\downarrow)$, Eq. (22) implies that $U_{z, z}<0$, making the $z$-axis the easy pseudospin orientation axis. Again, at $r_{\Delta}=1$ our model reduces to that of a single layer 2D systems whose easy-axis anisotrepy at even filling factors has been identified previously 10 In finitethickness single quantum wells, a QHF with pseudospin LLs of the same subband but different real-spin and orbitradius indices is also easy-axis. The magnitude of the anisotropy will decrease with layer thickness, as can be seen by comparing $U_{z, z}$ in (22) calculated for $r_{\Delta}=1$ and $r_{\Delta}=0$. (Note that the single-subband unbiased double well with finite tunneling, i.e. $r_{\Delta}=0$, models a single layer system with an effective thickness $d$.)

We now turn to the crossing of LLs with different subband indices, for which the pseudospin anisotropy physics is richer. In Fig. 3 we show examples of $n(\uparrow$ )$=n(\downarrow)$ bilayer QHFs for $\nu=1$ and $\nu=2$ based on same real-spin and opposite real-spin LLs respectively. Eqs. (10), (13),(17),(18), and Table Iimply four non-zero anisotropy terms for $n(\uparrow)=n(\downarrow) \equiv n$ and $s(\uparrow)=s(\downarrow)$ :

$$
\begin{aligned}
& U_{z, z}=u r_{\Delta}^{2}, \\
& U_{x, x}=u\left(1-r_{\Delta}^{2}\right), \\
& U_{x, z}=U_{z, x}=u r_{\Delta}\left(1-r_{\Delta}^{2}\right)^{1 / 2},
\end{aligned}
$$

where 


$$
u=\frac{d}{2}-\frac{1}{2} \int_{0}^{\infty} d q e^{-q^{2} / 2}\left[L_{n}\left(q^{2} / 2\right)\right]^{2}\left(1-e^{-d q}\right) .
$$

First term in the expression for energy $u$ comes from the Hartree interaction, the second term represents exchange contribution which is always smaller than the Hartree energy in this case, i.e., $u>0$. For zero tunneling between layers $\left(r_{\Delta}=1\right)$, the only non-zero anisotropy energy component, $U_{z z}=u$, is positive leading to the easyplane anisotropy of the QHF. In the absence of symmetry breaking fields (the linear pseudospin magnetization terms in (9)) the variational energy (9) is minimized when the pseudospins condense into a state magnetized at an arbitrary orientation within in the $x-y$ plane. In this state, electronic charge is distributed equally between the layers (pseudospin angle $\theta=0$ ) minimizing the electro static energy; spontaneous interlayer phase coherence the physical counter part of pseudospin order in this case, lowers the total energy of the system by strengthening interlayer exchange interactions.

For $r_{\Delta}^{2}<1$, Eqs. (23) imply the following quadratic terms in the HF total energy

$$
\sum_{i, j=x, y, z} U_{i, j} m_{i} m_{j}=u\left[r_{\Delta} m_{z}+\left(1-r_{\Delta}^{2}\right)^{1 / 2} m_{x}\right]^{2},
$$

i.e., the easy-plane is tilted from the $x-y$ plane in the pseudospin space by angle $\alpha=\arctan \left[\left(1-r_{\Delta}^{2}\right)^{1 / 2} / r_{\Delta}\right]$. The pseudospin basis states at different values of $r_{\Delta}$ are related, however, by a unitary transformation, which corresponds precisely to a rotation about $y$-axis by angle $-\alpha$, as seen from Eq. (11). The easy-plane where the above anisotropy energy is constant is, for any value of $r_{\Delta}$, the plane of equal charge per layer.

When pseudospin-up and pseudospin-down states differ by more than their subband indices, by their spin indices for example, the two pseudospin basis sates at different $r_{\Delta}$ are not related by a unitary transformation. In this case the magnetic anisotropy does depend on $r_{\Delta}$. For example, consider the case $\xi(\uparrow)=-\xi(\downarrow)$, $n(\uparrow)=n(\downarrow)$, and $s(\uparrow)=-s(\downarrow)$. For opposite real-spin LLs, all anisotropy terms that include pseudospin nonconserving scattering processes drop out. The only nonzero energy term, $U_{z, z}$, has the same value as in 23.). Hence, the QHF is isotropic in the unbiased $\left(r_{\Delta}=0\right)$ bilayer system while applying external bias $\left(r_{\Delta}>0\right)$ leads to easy-plane anisotropy in pseudospin space.

At this point let us make an experimentally important comment on the bilayer systems realized in wide single quantum wells. 20.199 The difference between this sample geometry and the double quantum well with narrow (in our model infinitely narrow) layers is in the nature of the barrier responsible for the bilayer character of the electropic system. In wide quantum wells the barrier is soft, 19 originating from Coulomb interactions among electrons in the well. Then the tunneling probability between layers is strongly dependent on the electron density and quantum well subband populations. This tunability makes wide single quantum wells an experimentally attractive alternative to double wells in studies of bilayer quantum Hall phenomena. The softness of the barrier can, however, lead to qualitatively important consequences for the ordered many-particle states. Translated into the pseudospin language, $\Delta_{t}$ cannot be treated as an external one-body field acting on the pseudospin particles but, in general, will depend11 on the pseudospin orientation in the ordered ground state. For the pseudospin LLs discussed in the previous paragraph $(\xi(\uparrow)=-\xi(\downarrow)$, $n(\uparrow)=n(\downarrow)$, and $s(\uparrow)=-s(\downarrow))$ and for $r_{\Delta}=0$, this effect can lead to an anisotropic QHF. LSDA calculations indicate that at low electron densities the anisotropy will be easy-plane while easy zxis anisotropy is more likely to develop at high densities 11 We make this remark to point out that not all results obtained for the double quantum well model are directly applicable to bilayers in wide single wells. In many cases the theoretical description of QHFs in wide single wells requires modifications of the idealized bilayer model to account for mixing of higher electrical subbands. The self-consistent LSDA for the growth direction single-particle orbitals is a particularly convenient, if somewhat ad hoc, method that allows any sample geometry to be studied while retaining the basic structure of the many-body HF formalism for QHFs.

In the remaining part of this section we consider pseudospin LLs with opposite subband indices and different orbit radius quantum numbers. At total filling factor $\nu=3$, for example, the pseudospin LLs will have the same real-spin while at $\nu=4$ opposite spin LLs can be aligned, as shown in Fig. 4. A common feature of the QHFs discussed below is the transition from a state with easy-axis anisotropy to a state with easy-plane anisotropy as $r_{\Delta}$ and the layer separation $d$ are varied. For $s(\uparrow)=s(\downarrow)$, Eqs. (10), (13), (17), (19), and Table 1 give three non-zero anisotropy energies

$$
\begin{aligned}
& U_{z, z}=\frac{r_{\Delta}^{2} d}{2}-\frac{1}{8} \int_{0}^{\infty} d q e^{-q^{2} / 2} \\
& \times\left\{\left[L_{n(\uparrow)}\left(q^{2} / 2\right)-L_{n(\downarrow)}\left(q^{2} / 2\right)\right]^{2}\left(1+e^{-d q}\right)\right. \\
& \left.+r_{\Delta}^{2}\left[L_{n(\uparrow)}\left(q^{2} / 2\right)+L_{n(\downarrow)}\left(q^{2} / 2\right)\right]^{2}\left(1-e^{-d q}\right)\right\}, \\
& U_{x, x}=U_{y, y}=-\frac{1}{4} \int_{0}^{\infty} d q e^{-q^{2} / 2} \frac{n_{<} !}{n_{>} !}\left(\frac{q^{2}}{2}\right)^{n_{>}-n_{<}} \\
& \times\left[L_{n<}^{n>-n_{<}}\left(q^{2} / 2\right)\right]^{2}\left(1-r_{\Delta}^{2}\right)\left(1-e^{-d q}\right),
\end{aligned}
$$

where

$$
n_{<} \equiv \min [n(\uparrow), n(\downarrow)], \quad n_{>} \equiv \max [n(\uparrow), n(\downarrow)] .
$$

The HF total energy contributions that are quadratic in the pseudospin magnetization components can be grouped as

$$
\sum_{i, j=x, y, z} U_{i, j} m_{i} m_{j}=\left(U_{z, z}-U_{x, x}\right) m_{z}^{2}+U_{x, x} .
$$


(Recall that $\hat{m}$ is a unit vector, i.e. $m_{x}^{2}+m_{y}^{2}=1-m_{z}^{2}$ ) From Eq. (26) we obtain that for $U_{z, z}-U_{x, x}<0$ the QHF has easy-axis anisotropy while for $U_{z, z}-U_{x, x}>0$ the system is an easy-plane ferromagnet. At the critical layer separation $d=d^{*}$, obtained from the condition $U_{z, z}=$ $U_{x, x}$, the magnetic anisotropy vanishes and a fine-tuned isotropy is achieved. It follows from Eqs. (25) that $d^{*}$ is finite for all values of $r_{\Delta}$.

For pseudospin LLs with $s(\uparrow)=-s(\downarrow)$, the anisotropy energy components $U_{x, x}$ and $U_{y, y}$ vanish and the critical layer separation $d^{*}$ corresponds to $U_{z, z}=0$, where $U_{z, z}$ is given by the same expression as in the $s(\uparrow)=s(\downarrow)$ case (see Eq. (25)). Since $U_{z, z}<0$ at $r_{\Delta}=0$, the critical separation $d^{*}$ diverges in the absence of external bias, i.e., easy-axis pseudospin anisotropy does not exist for any layer separation. For $r_{\Delta}>0$ the transition between easy-plane and easy-axis anisotropy occurs at finite $d$ as for the $s(\uparrow)=s(\downarrow)$ pseudospin LLs.

In Figs. 5(a) and (b) we show the magnetic anisotropy phase diagrams in the $d-r_{\Delta}$ plane calculated for $\nu=3$ and $\nu=4$ QHFs (see Fig. (1). Since the layer separation is in units of magnetic length, these figures imply that transition between easy-axis and easy-plane anisotropies at a given filling factor can be induced in one physical sample by changing the density of the $2 \mathrm{D}$ electron system. High electron densities would correspond to the easy-plane region, and low densities to the easy-axis region. Note that these numerical results confirm the general remark made above, since the critical layer diverges as $r_{\Delta} \rightarrow 0$ for $\nu=4$ while it remains finite for $\nu=3$.

\section{SYMMETRY BREAKING FIELDS}

The pseudospin orientation in a QHF ground state is determined by minimizing the variational total energy (9). In the absence of energy terms that are linear in pseudospin magnetization components, the HF ordered states spentaneously break continuous $\mathrm{SU}(2)$ or $\mathrm{U}(1)$ symmetryl in the case of isotropic or easy-plane QHFs respectively, and the discrete symmetry between pseudospin-up and pseudospin-down orientations in the case of easy-axis QHFs. In this section we take into account external and internal potentials which contribute to the linear terms in the HF total energy and comment on the pseudospin reversal that can be triggered by adjusting these symmetry breaking fields. We focus on an case which we feel is particularly appropriate for experimental study by considering the ordered $\nu=3$ quantum Hall state. Similar considerations would apply for all classes of QHFs discussed in this paper.

The pseudospin LLs in the bilayer $\nu=3$ QHF (see Fig. (1) have opposite subband indices and orbit radius quantum numbers $n=0$ and $n=1$, respectively. We call the $[\xi=-1, n=0, s=+1 / 2]$ LL the pseudospin-up state and the $[\xi=1, n=1, s=+1 / 2]$ LL the pseudospindown state. With this definition, the one-body potentials in (9) can be written as

$$
\begin{aligned}
b_{z} & =-\frac{1}{2}\left[\left(\Delta_{V}^{2}+\Delta_{t}^{2}\right)^{1 / 2}-\hbar \omega_{c}+I_{F}-I_{H, z}\right], \\
b_{x} & =\frac{1}{2} I_{H, x} \\
b_{y} & =0 .
\end{aligned}
$$

The effective field $I_{F}$ is the difference between pseudospin-up and pseudospin-down particle exchange energy with electrons in the fully occupied $[\xi=1, n=$ $0, s=+1 / 2]$ LL, i.e,

$$
\begin{aligned}
I_{F} & =\frac{1}{2} \int_{0}^{\infty} d q e^{-q^{2} / 2}\left\{\frac{q^{2}}{2}\left[1+r_{\Delta}^{2}+\left(1-r_{\Delta}^{2}\right) e^{-d q}\right]\right. \\
& \left.-\left(1-r_{\Delta}^{2}\right)\left(1-e^{-d q}\right)\right\} .
\end{aligned}
$$

For $r_{\Delta}>0$, electrons in the $[\xi=1, n=0, s= \pm 1 / 2]$ LLs produce also an electrostatic field, represented by $I_{H, z}$ and $I_{H, x}$ in (27), which screens the external bias potential. Since this effective field favors occupation of a particular layer rather than a particular pseudospin state it couples to $z$ and $x$ components of the pseudospin operator. The energy inbalance between the two layers produced by $\vec{I}_{H}$ is $2 d r_{\Delta}$ which, together with Eq. (1), gives

$$
\begin{aligned}
& I_{H, z}=2 d r_{\Delta}^{2} \\
& I_{H, x}=2 d r_{\Delta}\left(1-r_{\Delta}^{2}\right)^{1 / 2} .
\end{aligned}
$$

In the expression for the HF total energy (9), we included explicitly the contribution to the symmetry breaking fields which results from Coulomb interactions between electrons in the pseudospin LLs. For the $\nu=3$ QHF we are considering here, only $U_{\mathbf{1}, z}$ and $U_{z, \mathbf{1}}$ energies are non-zero:

$$
\begin{aligned}
U_{\mathbf{1}, z}=U_{z, \mathbf{1}} & =-\frac{1}{8} \int_{0}^{\infty} d q e^{-q^{2} / 2}\left[1+r_{\Delta}^{2}+\left(1-r_{\Delta}^{2}\right) e^{-d q}\right] \\
& \times\left(q^{2}-q^{4} / 4\right) .
\end{aligned}
$$

In the bilayer system with no tunneling $\left(r_{\Delta}=1\right)$, $I_{H, x}=0$ and the total symmetry breaking field, $b^{*} \equiv$ $b_{z}-U_{\mathbf{1}, z}$, is oriented along the $z$ pseudospin direction and is given by

$$
\begin{aligned}
b^{*} & =-\frac{1}{2}\left[\left(\Delta_{V}^{2}+\Delta_{t}^{2}\right)^{1 / 2}-\hbar \omega_{c}+\sqrt{\pi / 2} / 2-2 d\right. \\
& -\sqrt{\pi / 2} / 8] .
\end{aligned}
$$

In Figs. 6(a)-(c) we plot the pseudospin evolution with effective field $b^{*}$ for the three anisotropy regimes of a $\nu=3 \mathrm{QHF}$ with $r_{\Delta}=1$. At the phase boundary between easy-axis and easy-plane anisotropies, the $\nu=3$ QHF is isotropic and the pseudospin reverses abruptly at $b^{*}=0$ (see Fig. $6($ a)). In the easy-plane anisotropy 
regime, $\left(U_{z, z}-U_{x, x}>0\right)$, the pseudospin evolves continuously with $b^{*}$ as illustrated in Fig. 6(b) reaching alignment with $b^{*}$ at $\left|b^{*}\right| \geq U_{z, z}-U_{x, x}$. For the easy-axis anisotropy case $\left(U_{z, z}-U_{x, x}<0\right)$, the HF energy has two local minima at $m_{z}= \pm 1$ when $\left|b^{*}\right|<\left|U_{z, z}-U_{x, x}\right|$. The pseudospin-up and pseudospin-down polarized states are separated by an energy barrier which results in the hysteretic pseudospin-reversal behavior shown in Fig. 6(c).

In bilayer systems with non-zero tunneling, pseudospin reversal follows a more complicated pattern in which the competition between $x$ and $z$ components of the symmetry breaking field plays an important role. In general, the pseudospin will rotate in the $x-z$ plane, i.e. $m_{x}=\sqrt{1-m_{z}^{2}}$. Since the derivative of the HF energy with respect to $m_{z}$ diverges at $m_{z}= \pm 1$ due to the $I_{H, x}$ term the pseudospin will never align completely with the $z$-axis when $r_{\Delta}<1$.

\section{SUMMARY}

In the strong magnetic field limit, the physics of high mobility two-dimensional electron systems is usually dominated by electron-electron interactions except at integer filling factors, where the single-particle physics responsible for the gap between Landau levels assumes the dominant role. When external parameters are adjusted so that two or more Landau levels simultaneously approach the chemical potential, the integer filling factor case is less exceptional, interaction effects are always strong, and uniform density broken symmetry ground states analogous to those in conventional ferromagnets are common. In this paper we have discussed how the nature of these states depends on the character of the nearly degenerate Landau levels. Our attention is restricted to the case where only two Landau levels are close to the chemical potential and we distinquish these crossing Landau levels by introducing a pseudospin degree of freedom.

Using Hartree-Fock variational wavefunctions, we are able to derive an explicit expression for the dependence of ground state energy on pseudospin orientation for crossing LLs with any combination of quantum well subband, orbit radius and real-spin degree-of-freedom quantum numbers. As in conventional magnetic systems, qualitative differences exist between the physical properties of isotropic (Heisenberg) systems with no dependence of energy on pseudospin orientation, easy-axis (Ising) systems with discrete prefereed pseudospin orientations, and (XY) easy-plane systems for which the minimum is achieved simultaneously for a plane of orientations. Our mean-field results predict which class of pseudospin quantum Hall ferromagnet occurs in different circumstances. We focus on a model commonly used for bilayer quantum Hall systems in which the finite width of both quantum wells is neglected. The external parameters of the model are the Zeeman coupling strength, the bias potential be- tween the wells $\Delta_{V}$, and the single particle splitting due to interlayer tunneling $\Delta_{t}$. In the limit $\Delta_{t}=0$, this model applies to a single quantum well when the crossing Landau levels have the same subband wavefunction, i.e., are in the same quantum well. Classification predictions for this model as a function of layer separation $d$ and the ratio $r_{\Delta}=\Delta_{V} /\left(\Delta_{V}^{2}+\Delta_{t}^{2}\right)^{1 / 2}$ are summarized in the diagram 7 .

For the single quantum well case we find isotropic behavior when the orbit radius quantum numbers of the crossing Landau levels are identical, and easy-axis behavior otherwise. In general when the crossing Landau levels have identical subband wavefunctions, the nature of the pseudospin anisotropy does not depend on the paramters $d$ and $r_{\Delta}$. For different subband wavefunctions the pseudospin anisotropy can vary in the $d-r_{\Delta}$ plane. Particularly intriqueing is the case of crossing LLs with different subband and orbit radius quantum numbers where, at a given filling factor, the system can undergo a quantum phase transition from an easy-axis to easy-plane QHF. At the phase boundary the pseudospin anisotropy vanishes and a fine-tuned isotropy is achieved. The critical values of $d$ and $r_{\Delta}$ are experimentally accesible and may be accompanied by observable changes in pseudospin reversal properties as external parameters are varied.

\section{ACKNOWLEDGMENTS}

This work was supported by the National Science Foundation under grants DMR-9623511, DMR-9714055, and DGE-9902579 and by the Grant Agency of the Czech Republic under grant 202/98/0085.

${ }^{1}$ A.H. MacDonald, in Proceedings of the 1994 Les Houches Summer School on Mesoscopic Quantum Physics, edited by E. Akkermans et al. (Elsevier Science, Amsterdam, 1995), pp. 659-720.

${ }^{2}$ For a review on QHFs at $\nu=1$ see experimental chapter by J.P. Eisenstein and theoretical chapter by S.M. Girvin and A.H. MacDonald in Perspectives on Quantum Hall Effects (Wiley, New York, 1997).

${ }^{3}$ C. Kallin and B.I. Halperin, Phys. Rev. B 30, 5655 (1984); D.H. Lee and C.L. Kane, Phys. Rev. Lett. 64, 1313 (1990); S.L. Sondhi, A. Karlhede, S.A. Kivelson, and E.H. Rezayi, Phys. Rev. B 47, 16419 (1993); A.H. MacDonald, H.A. Fertig, and L. Brey, Phys. Rev. Lett. 76, 2153 (1996).

${ }^{4}$ S.E. Barrett, G. Dabbagh, L.N. Pfeiffer, and K.W. West, Phys. Rev. Lett. 74, 5112, (1995); R. Tycko, S.E. Barrett, G. Dabbagh, L.N. Pfeiffer, and K.W. West, Science 268, 1460 (1995); A. Smeller, J.P. Eisenstein, L.N. Pfeiffer, and K.W. West, Phys. Rev. Lett. 75, 4290 (1995); D.K. Maude, M. Potemski, J.C. Portal, M. Henini, L. Eaves, G. Hill, and 
M.A. Pate, Phys. Rev. Lett. 77, 4604 (1996); E.H. Aifer, B.B. Goldberg, and D.A. Broido, Phys. Rev. Lett. 76, 680 (1996); V. Bayot, E. Grivei, J.M. Beuken, S. Melinte, and M. Shayegan, Phys. Rev. Lett. 76, 4584 (1996); 79, 1718 (1997).

5 A.H. MacDonald, P.M. Platzman, G.S. Boebinger, Phys. Rev. Lett. 65, 775 (1990);

${ }^{6}$ T. Chakraborty and Pietiläinen, ibid 59, 2784 (1987); X.G. Wen and A. Zee, Phys. Rev. B 47, 2265 (1993); Z.F. Ezawa and A. Iwazaki, Int. J. Mod. Phys. B 19, 3205 (1992); L. Brey, Phys. Rev. Lett. 65, 903 (1990); H.A. Fertig, Phys. Rev. B 40, 1087 (1989); O. Narikiyo and D. Yoshioka, J. Phys. Soc. Jap. 62, 1612 (1993); R. Côté, L. Brey, and A.H. MacDonald, Phys. Rev. B 46, 10239 (1992); X.M. Chen and J.J. Quinn, ibid. 45, 11054 (1992); K. Moon et al., Phys. Rev. B 51, 5138 (1995); K. Yang et al., ibid. 54, 11644 (1996).

${ }^{7}$ I.B. Spielman, J.P. Eisenstein, L.N. Pfeiffer, and K.W. West, cond-mat/0002387.

${ }^{8}$ S.Q. Murphy, J.P. Eisenstein, G.S. Boebinger, L.N. Pfeiffer, and K.W. West, Phys. Rev. Lett. 72. 728 (1994);

9 T.S. Lay, Y.W. Suen, H.C. Manoharan, X. Ying, M. Santos, and M. Shayegan, ibid. 50, 17725 (1994); M. Abolfath, R. Golestanian, and T. Jungwirth, Phys. Rev. B 61, 4762 (2000).

10 T. Jungwirth, S.P. Shukla, L. Smrčka, M. Shayegan, and A.H. MacDonald, Phys. Rev. Lett. 81, 2328 (1998).

${ }^{11}$ V. Piazza, V. Pellegrini, F. Beltram, W. Wescheider, T. Jungwirth, and A.H. MacDonald, Nature 402, 638 (1999).

12 G.F. Giuliani and J.J. Quinn, Phys. Rev. B31, 6228 (1985).

${ }^{13}$ Recently observed hysteresis at $\nu=2 / 5$ and $4 / 9$, corresponding to composite fermion filling factors $\nu=2$ and 4 , has also been interpreted within the easy-axis QHF framework: H. Cho, J.B. Young, W. Kang, K.L. Campman, A.C. Gossard, M. Bichler, and W. Wescheider, Phys. Rev. Lett. 81, 2522 (1998).

${ }^{14}$ L. Zheng, R.J. Radtke, and S. Das Sarma, Phys. Rev. Lett. 78, 2453 (1997); S. Das Sarma, S. Sachdev and L. Zheng, Phys. Rev. Lett. 79, 917 (1997); Phys. Rev. B 58, 4672 (1998); L. Brey, E. Demler, S. Das Sarma, Phys. Rev. Lett. 83, 168 (1999); B. Paredes, C. Tejedor, L. Brey, and L. Martín-Moreno, Phys. Rev. Lett. 83, 2250 (1999).

${ }^{15}$ V. Pellegrini, A. Pinczuk, B.S. Dennis, A.S. Plaut, L.N. Pfeiffer, and K.W. West, Phys. Rev. Lett. 78, 310 (1997); Science 281, 799 (1998); A. Sawada, A.F. Ezawa, H. Ohno, Y. Horikoshi, Y. Ohno, S. Kishimoto, F. Matsukura, Phys. Rev. Lett. 80, 4534 (1998).

${ }^{16}$ G. Bastard, Wave Mechanics Applied to Semiconductor Heterostructures (Les Éditions de Physique, Paris, 1990).

17 S.J. Papadakis, E.P. De Poortere, and M. Shayegan, Phys. Rev. B 59, R12743 (1999).

${ }^{18}$ K.J. Goldammer, S.J. Chung, W.K. Liu, M.B. Santos, J.L. Hicks, S. Raymond, and S.Q.Murphy, J. Cryst. Growth 202, 753 (1999).

19 Y.W. Suen, J. Jo, M.B. Santos, L.W. Engel, S.W. Hwang, and M. Shayegan, Phys. Rev. B 44, 5947 (1991); T.S. Lay, T. Jungwirth, L. Smrčka, M. Shayegan, Phys. Rev. B 56, R7092 (1997).

\begin{tabular}{ccccc} 
& $\mathbf{1}$ & $x$ & $y$ & $z$ \\
$\mathbf{1}$ & $B_{1}^{+}+B_{2}^{+}$ & $B_{5}^{-}+B_{6}^{+}$ & $\frac{1}{i} B_{5}^{-}+\frac{1}{i} B_{6}^{-}$ & $B_{1}^{-}-B_{2}^{-}$ \\
$x$ & $B_{7}^{+}+B_{8}^{+}$ & $B_{3}^{+}+B_{4}^{+}$ & $\frac{1}{i} B_{3}^{-}-\frac{1}{i} B_{4}^{-}$ & $B_{7}^{+}-B_{8}^{+}$ \\
$y$ & $\frac{1}{i} B_{7}^{-}+\frac{1}{i} B_{8}^{-}$ & $\frac{1}{i} B_{3}^{-}+\frac{1}{i} B_{4}^{-}$ & $B_{3}^{+}-B_{4}^{+}$ & $\frac{1}{i} B_{7}^{-}-\frac{1}{i} B_{8}^{-}$ \\
$z$ & $B_{1}^{-}+B_{2}^{-}$ & $B_{5}^{+}-B_{6}^{+}$ & $\frac{1}{i} B_{5}^{-}-\frac{1}{i} B_{6}^{-}$ & $B_{1}^{+}-B_{2}^{+}$ \\
\hline \hline
\end{tabular}

TABLE I. Coulomb interaction matrix elements $W_{i, j}$; $i, j=\mathbf{1}, x, y, z$. The $B_{n}^{ \pm}$terms are defined in (8).

FIG. 1. Schematic of the conduction band edge profile of a biased double-quantum-well sample with non-zero tunneling between 2D layers. Energy levels due to quantization of electron motion along the growth direction are also indicated. For concrete calculations we assume infinitely narrow quantum wells separated by the distance $d$.

FIG. 2. Schematic LL diagrams for $\nu=1$ (a) and $\nu=2$ (b) single-subband QHFs. Index $\sigma=\uparrow, \downarrow$ labels the pseudospin LLs. The crossing LLs are indicated by half-solid dots, while inert filled LLs are indicated by solid dots and inert empty LLs by open dots.

FIG. 3. Schematic LL diagrams for $\nu=1$ (a) and $\nu=2$ (b) bilayer QHFs. The pseudospin LLs have opposite subband indices and same (a) or opposite (b) real-spins.

FIG. 4. Schematic LL diagrams for $\nu=3$ (a) and $\nu=4$ (b) bilayer QHFs. The pseudospin LLs have opposite subband indices, different orbit radius quantum numbers and same (a) or opposite (b) real-spins.

FIG. 5. Magnetic anisotropy phase diagrams for bilayer $\nu=3$ (a) and $\nu=4$ (b) QHFs from Fig. 1. In the white region the anisotropy is easy-axis, in the grey region the QHF has easy-plane anisotropy. At the phase boundary the ferromagnetic state is isotropic.

FIG. 6. Pseudospin orientation as a function of the effective field $b^{*}$ for the isotropic QHF and as a function of $b^{*}$ relative to the anisotropy energy $\left|U_{z, z}-U_{x, x}\right|$ for the easy-plane (b), and easy-axis (c) QHFs at $\nu=3$.

FIG. 7. Magnetic anisotropy of QHFs with pseudospin LL subband indices $\xi(\sigma)$, orbit radius quantum numbers $n(\sigma)$, and real-spins $s(\sigma)$. 
b)

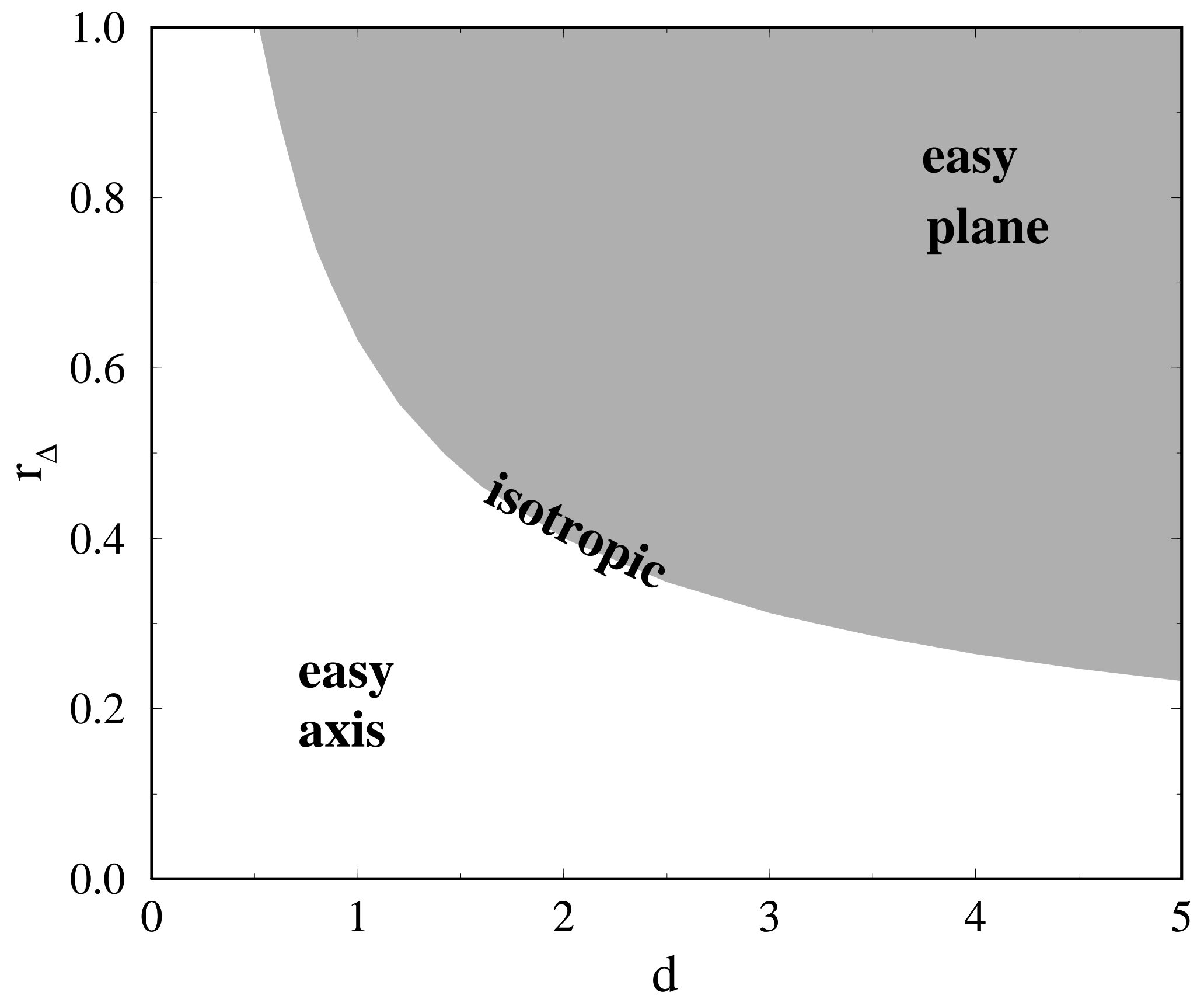



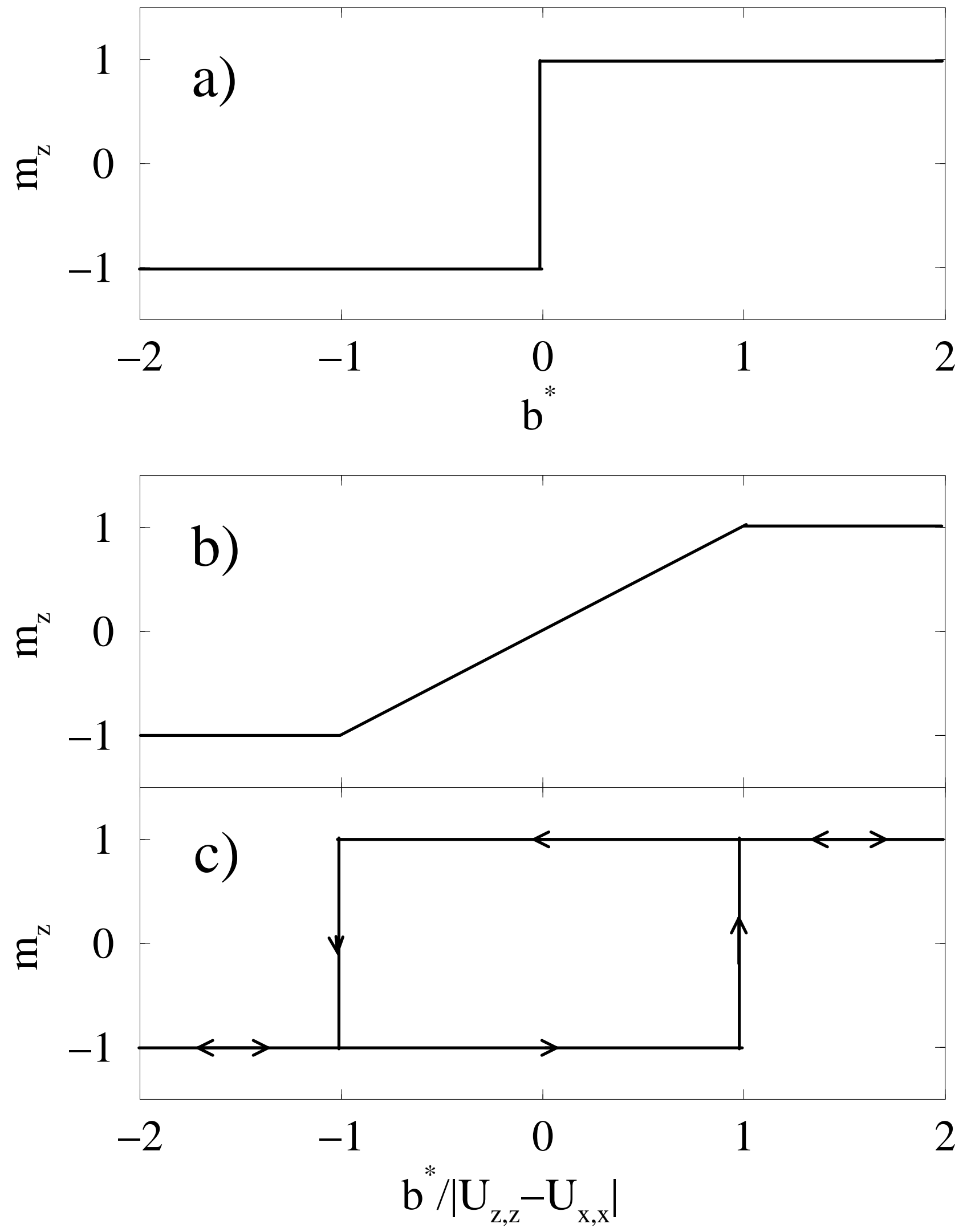